treat pharyngeal infections caused by gonococci with an $\mathrm{MIC} \leq 0.5$ $\mu \mathrm{g} / \mathrm{mL}$. A regimen of $800 \mathrm{mg}$ orally every 8 hours for three doses would achieve total levels high enough to treat gonococci with an MIC $\leq 1.0 \mu \mathrm{g} / \mathrm{mL}$. Free cefixime levels attained with $800 \mathrm{mg}$ of cefixime every 12 hours for two doses would exceed $0.5 \mu \mathrm{g} / \mathrm{mL}$ for over 24 hours; $800 \mathrm{mg}$ every 6 hours for three doses would achieve free levels that exceed $1.0 \mu \mathrm{g} / \mathrm{mL}$ for nearly 24 hours.

Conclusion Two-to-three $800 \mathrm{mg}$ doses of cefixime could be an effective therapy for pharyngeal infections with gonococci with cefixime MICs of $0.5-1.0 \mu \mathrm{g} / \mathrm{mL}$. A pharmacokinetics trial to evaluate the accuracy of these simulations and the safety and tolerability of the proposed regimens is currently underway.

\section{P2.091 A REPEATED LOW DOSE CO-CHALLENGE MODEL OF SHIV- RT AND HSV-2 IN RHESUS MACAQUES}

doi:10.1136/sextrans-2013-051184.0355

'J Kenney, 'N Derby, 'M Aravantinou, 'S Rana, ${ }^{2} \mathrm{~J} D$ Lifson, M Piatak, ${ }^{2} \mathrm{Jr},{ }^{3} \mathrm{~A}$ Gettie, 4J Blanchard, 'M Robbiani. 'Population Council, New York, NY, United States, ${ }^{2}$ SAICFrederick, Inc., NCl Frederick, Frederick, MD, United States, ${ }^{3}$ Aaron Diamond AIDS Research Center, New York, NY, United States, ${ }^{4}$ Tulane National Primate Research Center, Covington, LA, United States

Background HIV acquisition is facilitated by HSV-2 infection, making microbicides that block both viruses desirable for limiting HIV transmission. We have tested microbicides in a stringent efficacy model: vaginal co-challenge with a single high dose of SHIVRT $\left(10^{3} \mathrm{TCID}_{50}\right)$ and HSV-2 $\left(2 \times 10^{8} \mathrm{pfu}\right)$ in DepoProvera (DP)-treated macaques. Here we established a model mimicking real world exposure: repeated low dose SHIV/HSV-2 co-challenge in non-DPtreated animals.

Methods Two groups of macaques were co-challenged weekly for 11 wks with SHIV (10 or $\left.50 \mathrm{TCID}_{50}\right)$ and HSV-2 (10 pfu) after which the SHIV dose was increased to 200 TCID $_{50}$ in all animals for 9 more co-challenges. HSV-2 shedding in vaginal swabs and SHIV plasma viremia were determined. Antibodies (Abs) to SIV and HSV2, HSV-2-specific T cell responses and the hormones estradiol and progesterone were measured in the blood.

Results After 11 co-challenges, SHIV infections were detected in $1 / 3$ animals from the 10 TCID $_{50}$ SHIV group and $1 / 3$ from the 50 TCID $_{50}$ SHIV group (after 2 and 8 challenges, respectively). Upon increasing the SHIV dose, two more animals became infected (after 1 and 5 more co-challenges), but the last two remained uninfected. SHIV viremia was similar in all infected animals, which all developed SIV-specific Abs. All animals (6/6) became HSV-2 infected. Initial analyses suggest that the frequency of HSV-2 shedding was greater in non-DP-treated animals repeatedly exposed to $10^{7} \mathrm{pfu}$ than we previously observed for DP-treated animals that received a single $2 \times 10^{8}$ pfu dose of HSV-2 with SHIV ( $\left.p<0.0001\right)$. HSV2 -specific IgG responses were not detected; $\mathrm{T}$ cell responses are being analysed.

Conclusion We have developed a repeated low dose co-challenge model to evaluate microbicides against SHIV and HSV-2. SHIV infection frequency was $67 \%$ in this model, similar to the single high dose co-challenge. HSV-2 infection was enhanced compared to the single high dose model.

\section{P2.092 IN VITRO AND IN VIVO EVALUATION OF CARRAGEENAN- BASED FORMULATIONS TO PREVENT HPV ACQUISITION}

doi:10.1136/sextrans-2013-051184.0356

'A Rodriguez, '0 Mizenina, 'L Kizima, 'K Levendosky, 'K Kleinbeck, 'N Derby, 'M Robbiani, ${ }^{2} \mathrm{~B}$ Herold, 'T Zydowsky, 'J Fernandez-Romero. 'Population Council, New York, NY, United States; ${ }^{2}$ Albert Einstein College of Medicine, Bronx, NY, United States
Background Human papillomavirus (HPV) constitutes one of the major sexually transmitted viral infections. Vaccines against HPV are commercially available but vaccination rates are currently low around the world due to parental autonomy, three dose regimen, cost and the need for cold chain storage. In vitro and in vivo data have indicated that carrageenan (CG)-based microbicide formulations may prevent HPV infection but additional data is needed to assess the durability of this antiviral activity and the effect of biological fluids.

Methods A proprietary 3\% CG gel formulation (Population Council) and the commercial sexual lubricant Divine 9 were tested for their anti-HPV activity against HPV16, 18, and 45 pseudoviruses (PsVs). The anti-HPV PsV activity was estimated using the in vitro luciferase assay in HeLa cells and $\mathrm{IC}_{50}$ values were calculated using a dose-response-inhibition analysis on GraphPad Prism v5.0 software. The HPV PsV luciferase mouse model was performed to test the in vivo activity of the gels. The formulations were applied intravaginally in a BAT24 $(-2 \mathrm{~h} /+2 \mathrm{~h})$ dosing regimen or in a single $-24 \mathrm{~h}$ application before challenging with HPV16 or $45 \mathrm{PsV}$ in PBS or seminal plasma. In vivo luciferase expression was measured $24 \mathrm{~h}$ later and the Mann Whitney $\mathrm{U}$ test $(\mathrm{P}<0.05)$ was used for statistical analyses.

Results Both CG and Divine 9 showed broad-spectrum anti-HPV activity in vitro $\left(\mathrm{IC}_{50}: 1-20 \mathrm{ng} / \mathrm{ml}\right)$ and significantly decreased HPV $\mathrm{PsV}$ infection in the mouse model; the in-house formulation afforded better protection than Divine 9 in the BAT24 $(p=0.0101)$ or the single $-24 \mathrm{~h}$ application $(\mathrm{p}=0.0008)$ dosing regimens. CG formulations retained full activity in the murine model when PsVs were mixed with human seminal plasma.

Conclusions The potential broad-spectrum activity of CG formulations and the durability of protection, even in the presence of seminal plasma, supports further advancement of CG to prevent HPV acquisition.

\section{P2.093 ROLE OF 5\% KOH SOLUTION FOR THE TREATMENT OF GENITAL MOLLUSCUM CONTAGIOSUM IN ADULT FEMALE PATIENTS}

doi:10.1136/sextrans-2013-051184.0357

\section{A D Sharma. Dermacare Clinic, Bongaigaon, India}

Background To evaluate the role of $5 \% \mathrm{KOH}$ solution in the treatment of genital Molluscum contagiosum (MC) in adult female patients.

Methods All total 19 female patients with multiple genital MC were included for this study. All the patients were seronegative for the human immunodeficiency virus. Pregnant and lactating woman were not included in the study. It was a clinic based study. $5 \% \mathrm{KOH}$ solution was applied to the skin lesions with swab stick. After 5 minutes skin lesions were washed with cold water gauge sponge. The procedure was repeated at 4 days interval. Patients were evaluated at the end of every 5 sittings and results were recorded.

Results 5 patients had complete clearance of their skin lesions by the end of 5 sittings. Another 11 patients had complete clearance of their skin lesions by the end of 10 sittings. Remaining patients had complete clearance of their skin lesions by the end of 15 sittings. Side effects of the treatment noted in the study were very few and very mild in nature

Conclusion This study showed that $5 \% \mathrm{KOH}$ solution is a safe, inexpensive \& effective clinic procedure for the treatment of genital $\mathrm{MC}$ in adult female patients. It is a simple technique with wide patient acceptance and with excellent cosmetic results. Side effects of the treatment noted in the study was very mild and well tolerated by the patients. 